\title{
TIC para la orientación académica y profesional en Educación Secundaria: El programa Orienta
}

\author{
Delia García Arias. Universidad de Jaen \\ Recepción: 08.10.2019 | Aceptado: 07.11.2019 \\ Correspondencia a través de ORCID: Delia García Arias \\ iD 0000-0002-5001-2718 \\ Citar: García Arias, D. (2019). TIC para la orientación académica y profesional en Educación Secundaria: \\ El programa Orienta. REIDOCREA, 8, 375-388.
}

\begin{abstract}
Resumen: Este trabajo aborda el uso de una herramienta TIC, el programa Orienta, como apoyo a la labor de la orientación académica y profesional en los centros de educación secundaria de Granada. El estudio se centra en la evaluación y valoración de esta herramienta por parte de una muestra de orientadores educativos de la provincia de Granada. A partir de sus respuestas se pretende obtener información valiosa que permita identificar y plantear, si fuera necesario, propuestas de mejora de dicha herramienta. Con este artículo se pretende mejorar la intervención de los orientadores en el ámbito académico y profesional a través de un recurso TIC específico para su labor, con el fin último de proporcionar al alumnado una orientación de calidad, individualizada y adaptada a sus necesidades y singularidades que les ayude a tomar decisiones en su proyecto laboral y de vida y les permita desarrollar todo su potencial, tanto a nivel personal como profesional.
\end{abstract}

Palabras clave: Orientación académica y profesional | Herramientas TIC

ITC tool for the academic and professional orientation in High Schools: 'Orienta program'

\begin{abstract}
This research project addresses how the ITC tool called 'Orienta program' supports the efforts of the academic and professional orientation across secondary study centres in the province of Granada (Spain). This research focuses on the feedback given on this ITC tool by a selected sample of professional career advisors of the abovementioned province. Their answers will provide insights that would allow us to identify and consider proposals to improve this tool, if appropriate. This research aims to enhance the academic and professional guidance processes by means of an ITC resource specifically designed for that purpose, having in mind the ultimate goal is to provide students with an individualised, high-quality orientation tailored to their needs and singularities that helps in their career and life project decision-taking processes and allows them to achieve their full personal and professional potential.
\end{abstract}

Keywords: Academic and professional orientation | ITC tools

\section{Introducción}

Este trabajo se focaliza específicamente en la labor de orientación académica y profesional que desarrollan los orientadores educativos en los centros de educación secundaria de Granada teniendo en cuenta la realidad educativa actual y su relación con las herramientas TIC. Se ha desarrollado la investigación en un ámbito del que formo parte como profesional de la orientación, cuerpo profesional en el que desarrollo mi labor docente en la actualidad.

Este artículo se centra en la orientación tanto desde el ámbito académico como profesional y tiene como punto de partida fundamental la necesidad que presentan todos los alumnos en un momento específico de su vida, cuando están a punto de terminar sus estudios de Educación Secundaria Obligatoria (de ahora en adelante, ESO) y Bachillerato: la importante decisión de qué hacer con respecto a su futuro académico y profesional. Esta cuestión genera incertidumbre y un fuerte sentimiento de desazón e intranquilidad en el alumnado y es una de las principales preocupaciones que trasladan a los orientadores. Para intentar dar respuesta a esta necesidad, el presente trabajo examinará cómo las TIC pueden ser una herramienta importante y necesaria en este proceso. 
Esta investigación se divide en tres fases. La primera fase se centra en el desarrollo de un cuestionario destinado a los orientadores educativos con el objetivo de recoger información sobre la opinión y la valoración que tienen estos profesionales sobre los distintos aspectos del programa Orienta. En la segunda fase se pretenden extraer conclusiones sobre la funcionalidad y la eficacia de este programa a partir de la información y las respuestas extraídas, con el fin de intentar mejorar aquellos aspectos que lo precisen, si fuera el caso. Por último, con estas conclusiones, se presentarán varias propuestas de mejora técnica, comunicativa y didáctica del programa, que pueden servir de base para mejorar en un futuro la herramienta objeto de estudio, el programa TIC Orienta.

Los recursos TIC y, en concreto, el programa Orienta, son muy importantes en cuanto sirven de apoyo a la labor de los orientadores. De acuerdo con Sánchez (2017), las TIC permiten a los orientadores enfocar sus actuaciones a elementos más significativos del proceso orientador, ya que les permite reducir la dedicación a otras tareas de tipo mecánico.

La orientación académica y profesional llevada a cabo en los centros educativos busca dar una respuesta a las demandas laborales de nuestra sociedad española actual, proporcionando un asesoramiento a los jóvenes sobre su futuro laboral y académico. Ahora bien, el orientador debe contar con conocimientos amplios y actualizados del mundo laboral y tener en consideración cuáles son las características del mercado laboral español para poder ajustar adecuadamente su intervención al alumnado, además de haber evaluado los intereses, motivaciones y aptitudes personales de cada uno de ellos.

Para poder tomar una decisión sobre su futuro, el alumnado tiene que conocer la situación del mercado laboral, es decir, tiene que tener información sobre los datos de desempleo, los porcentajes de titulados que han encontrado trabajo y qué carreras, ciclos formativos u otros tipos de formación cuentan con una tasa menor de desempleo. Esta información responde a la necesidad del alumnado de conocer la situación sobre el mundo laboral, cuáles son los puestos de trabajo más demandados y cuáles son las titulaciones que más salidas ofrecen. Por ejemplo, existen puestos de trabajo con una alta demanda de trabajadores, pero no hay suficientes personas para cubrirlos, mientras que hay puestos de trabajos con una gran saturación de personas que compiten por cubrirlos.

La situación actual del mercado laboral en España se caracteriza por una alta tasa de desempleo juvenil. La relación entre el mercado de trabajo y la población joven en España se ha caracterizado en los últimos años por una tendencia a la precariedad, y los últimos datos dejan patente la fragilidad del mercado laboral español. Según los datos del Observatorio de las Ocupaciones de 2016, en el año 2006, la tasa de desempleo juvenil alcanzó un 13,80 \%, y en 2012 ascendió a su valor máximo de los últimos 10 años con un 42,73 \%. Por su parte, Andalucía es una de las comunidades autónomas en España con una de las tasas de desempleo juvenil más altas del país. Según datos del Instituto Nacional de Estadística, el número de jóvenes desempleados menores de 25 años se situó en el primer trimestre de 2019 en 110,1 miles de personas. El número de jóvenes desempleados en Andalucía menores de 25 años está por encima de los jóvenes desempleados en toda la totalidad del territorio español.

De acuerdo con Abad, Gago y Merchante (2019), un elemento clave de la alta tasa de desempleo juvenil son los desajustes observados entre las habilidades requeridas para el desempeño de la ocupación profesional con la formación adquirida en la educación formal. Según los datos recogidos en la Encuesta de Población Activa, en el año 2015, 
un $68 \%$ de los ocupados menores de 25 años desempeñó un puesto laboral para el que se requerían menos habilidades de la que poseían. Y este problema se agravó aún más desde 2013 a 2016, ya que la sobrecualificación creció en más de un $12 \%$.

En el trasfondo de este fenómeno subyace la cuestión de cómo se produce la transición del aula al puesto de trabajo, es decir, cómo los alumnos con el itinerario académico que han realizado pueden encontrar un empleo que se ajuste a sus motivaciones, intereses y aptitudes. Esta cuestión está estrechamente relacionada con las funciones que debe cumplir el sistema educativo español y la orientación académica y profesional (Martínez, Pérez y Martínez, 2014), puesto que los orientadores de los centros educativos de secundaria cumplen una labor fundamental a la hora de ayudar al alumnado en la toma de decisiones respecto a su futuro profesional. Una correcta elección de un itinerario académico basado en las aptitudes y capacidades personales del alumnado, así como un conocimiento actualizado de la situación del mercado laboral, favorecerá que los alumnos elijan correctamente la profesión más adecuada para cada uno de ellos.

Por tanto, es muy importante que la acción y la intervención profesional en el ámbito de la orientación sean eficientes, ya que no solo va a favorecer el proyecto de vida futuro del alumno, sino que redunda en el beneficio de la sociedad en su conjunto. En una era digital como la nuestra, con una sociedad fuertemente influenciada por la tecnología de la información, las TIC se presentan como una herramienta clave para la mejora de los procesos de enseñanza-aprendizaje y de orientación que los orientadores pueden utilizar para ayudarles a realizar su labor.

\section{Objetivos o hipótesis}

El objetivo general que se persigue con este trabajo de investigación es explorar y analizar las posibilidades del programa Orienta en el ámbito de la orientación educativa en la etapa de educación secundaria (ESO y Bachillerato) para identificar y plantear, si fuera necesario, propuestas de mejora del mismo.

Este objetivo general se va a concretar en unos objetivos específicos relacionados entre sí, de modo que las intenciones de esta investigación queden delimitadas de una forma más precisa:

1. Conocer el grado de satisfacción de los diferentes aspectos que componen el recurso TIC, llamado programa Orienta, por parte de los orientadores educativos en la provincia de Granada con experiencia en la utilización de este recurso.

2. Plantear propuestas de mejora propias de los diversos elementos que componen el programa Orienta a partir de los datos recogidos de los orientadores participantes en la investigación.

\section{Métodos}

Para cumplir los objetivos planteados en esta investigación, la metodología empleada para la consecución de dichos objetivos es la modalidad cuantitativa de tipo descriptivo con un cuestionario para la recogida de información. De acuerdo con HernándezSampieri y Mendoza (2018): "Los estudios descriptivos pretenden especificar las propiedades, características y perfiles de personas, grupos, comunidades, procesos, objetos o cualquier otro fenómeno que se someta a un análisis" (p. 108). 


\section{Población y participantes}

La población a la que va dirigida este estudio son todos los profesionales de este campo que están trabajando actualmente en centros de educativos de enseñanza secundaria o aquellos que, aunque no estén en activo (interinos que hayan vuelto a la bolsa después de cubrir una baja), tengan experiencia en este ámbito de la educación en la provincia de Granada.

Respecto a los participantes de esta investigación, se ha realizado un muestreo por conveniencia, ya que no fue posible contar con la participación de todos los orientadores de la provincia granadina. En concreto, en este estudio han participado 38 orientadores educativos de dicha provincia que accedieron de manera voluntaria a responder al cuestionario.

\section{Instrumentos}

El instrumento que vamos a utilizar para recoger los datos en esta investigación es el cuestionario. Los cuestionarios son la herramienta más ampliamente usada en la investigación ya que cuenta con muchas ventajas, entre ellas, el hecho de que resulte ser una herramienta económica, anónima y la posibilidad de poder hacer las mismas preguntas a todos los sujetos, quedando así asegurada la homogeneidad (McMillan y Schumacher, 2005).

El cuestionario utilizado para recoger los datos de esta investigación está basado en un cuestionario previamente elaborado para la investigación "Plataformas de campus virtual con herramientas de software libre: Análisis comparativo de la situación actual en las universidades españolas" (Prendes, 2009). El cuestionario elaborado y utilizado en dicha investigación fue validado por un juicio de expertos y es de tipo cuantitativo. Este cuestionario se compone de ítems de preguntas cerradas y se ha articulado en torno a cinco grandes bloques. El cuestionario completo se puede consultar en el ANEXO I del presente trabajo.

\section{Procedimiento}

Una vez adaptado el cuestionario que se utilizó para la recogida de datos de la presente investigación, se procedió a trasladarlo a una versión online. Para ello se utilizó la herramienta "Formularios" de Google. De esta manera, la recogida de datos se realizó de manera totalmente virtual y anónima.

El enlace al cuestionario virtual se proporcionó a los 38 orientadores educativos participantes en este estudio, que se encuentran trabajando o han trabajado en este ámbito de la educación con anterioridad. El cuestionario fue respondido por los participantes de manera voluntaria y desinteresada.

\section{Resultados}

Tras el procedimiento de recogida de datos mediante el cuestionario respondido por orientadores educativos, se va a dar paso a la realización del análisis exhaustivo de estos datos, de manera que se expongan las valoraciones de los orientadores sobre el programa Orienta. 
A) Datos de los profesionales de la muestra participante

En el primer apartado del cuestionario, "datos del encuestado", se ha recogido información sobre sobre el perfil de los orientadores. La mayoría de los participantes en esta investigación trabaja o ha trabajado en institutos de educación secundaria (76 \%), mientras que el resto de los orientadores realizan o han realizado su labor profesional en equipos de orientación educativa (24 \%) (Figura 1).

Respecto a los años de experiencia como orientadores educativos, hay un amplio rango de tiempos de servicio. En el primer rango de tiempo, se sitúa el $24 \%$ de los orientadores educativos con experiencia entre 1 mes y 1 año. En el segundo intervalo, se encuentran los orientadores que tienen una experiencia de entre 1 y 5 años, que conforman el 54 $\%$ de los orientadores participantes. En el último lugar se encuentran los orientadores educativos con un tiempo de servicio de más de 5 años, los cuales conforman el $22 \%$.

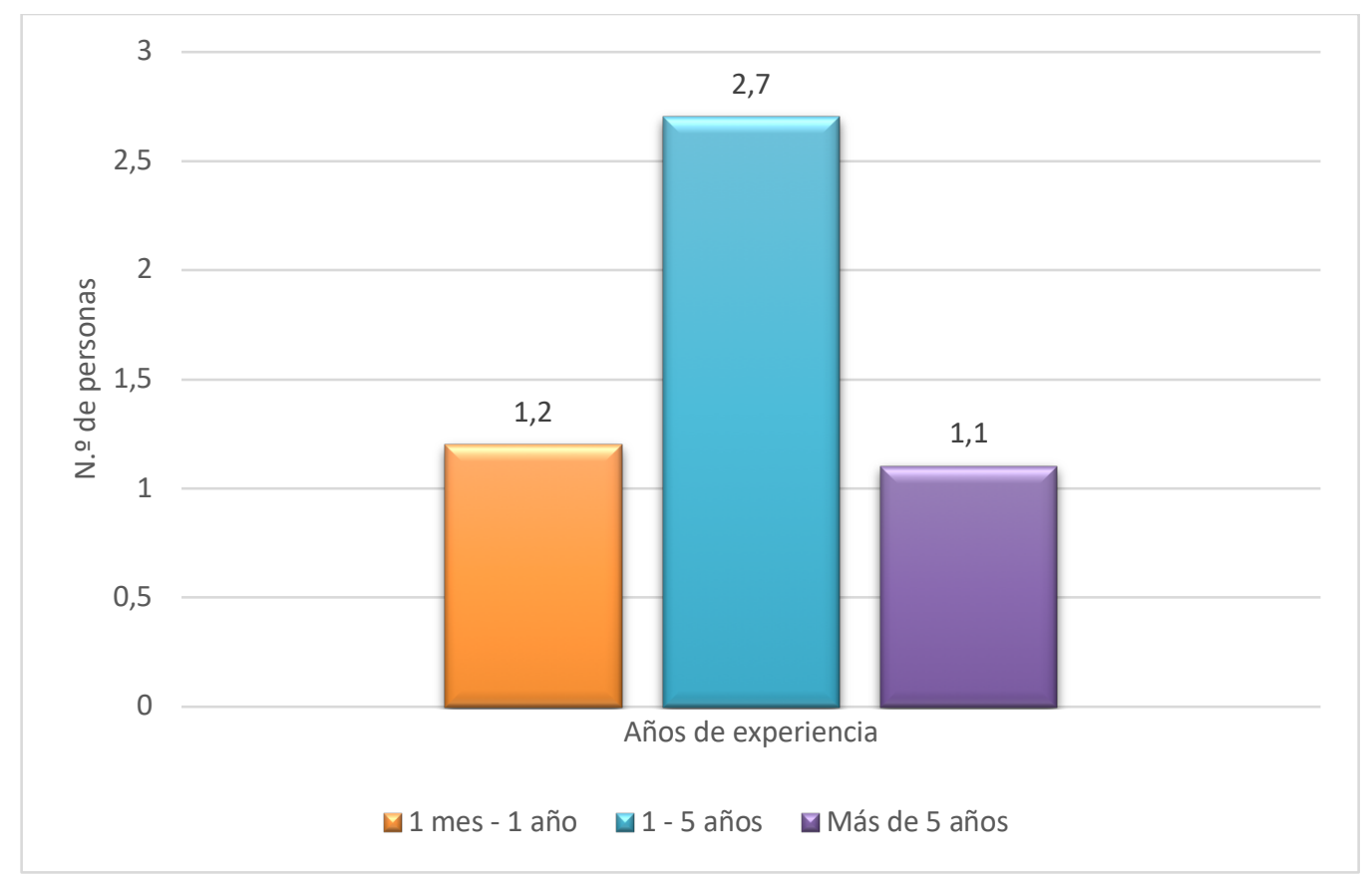

Figura 1. Gráfico: Apartado "Datos del encuestado" del cuestionario.

B) Aspectos generales

En el segundo gran bloque de ítems del cuestionario, llamado "aspectos generales", se agrupan un conjunto de seis ítems relacionados con esta temática (Figura 2). La mayor puntuación la ha obtenido el ítem "orientación del usuario dentro del programa", con una puntuación media de 4,9. En términos de alta puntuación, le sigue el ítem "facilidad de uso del programa", con un valor aritmético situado en 4,8. El ítem "facilidad de acceso a cualquier elemento del programa" obtiene un valor casi similar al anterior, con una media de 4,5 .

Los tres últimos ítems se sitúan en unos valores más bajos. El ítem "organización de la pantalla" obtiene una puntuación de 3,6. Le sigue el ítem "diseño gráfico (colores, letra, iconos, etc.)" con un valor de 3,2. Por último, el ítem que ha recibido el valor más bajo es "estética del programa", con un valor de 2,2. 


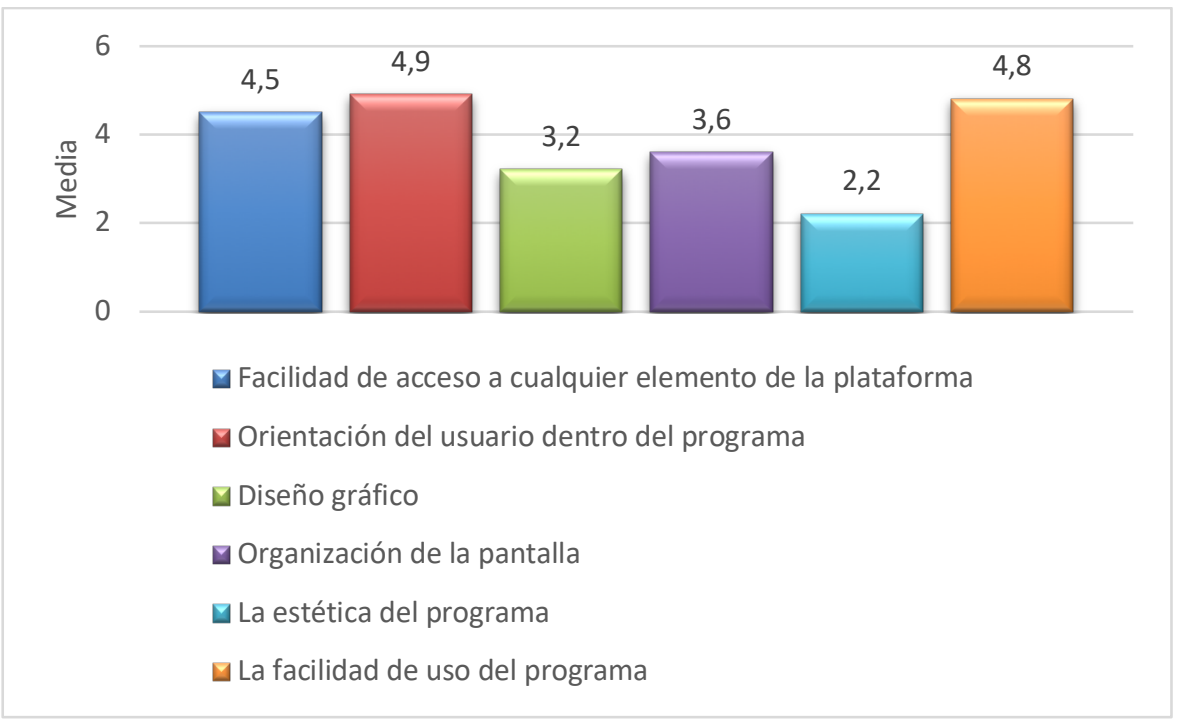

Figura 2. Gráfico: Apartado "Aspectos generales" del cuestionario.

\section{C) Comunicación}

En cuanto al conjunto de ítems agrupados en la categoría de "comunicación", los valores medios de estos ítems (Figura 3) son, por regla general, más bajos que los de la categoría anterior.

El ítem mejor puntuado en esta categoría es el de "blog del programa", con un valor medio de 4,3. Le sigue en términos de puntuación más alta, el ítem "guía de usuario/sistema de ayuda", con un valor medio de 4,1. El tercer ítem con mejor puntuación en esta categoría es el "correo de contacto con el administrador", con un 2,9.

El resto de ítems de esta categoría tienen, por contraste con los ítems anteriores, una puntuación muy baja. El ítem "calendario/agenda" tiene un valor medio de 2,3. Le sigue el ítem "tablón de anuncios", con un valor de 1,5. A continuación, la puntuación del ítem "uso de las redes sociales" es de 1,2. Por último, el ítem "foro del programa" recibe una valoración de 0,9.

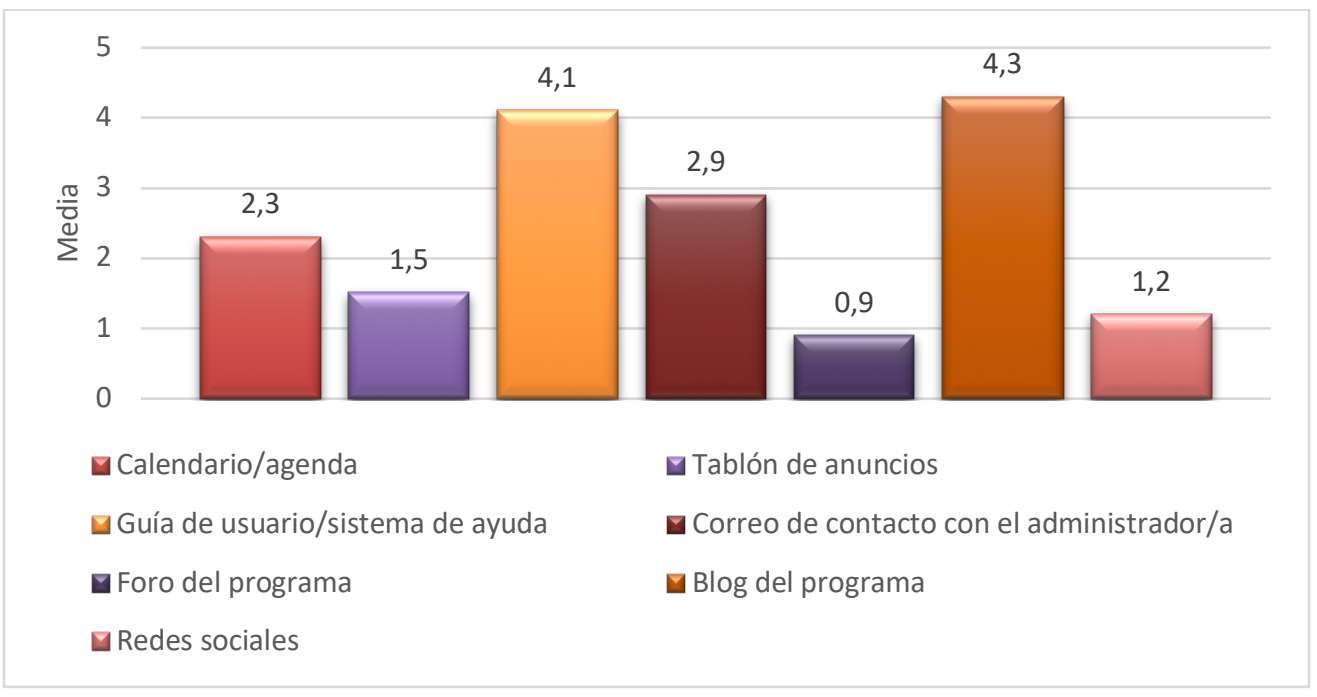

Figura 3. Gráfico: Apartado "Comunicación" del cuestionario. 


\section{D) Contenido didáctico}

En cuanto a los ítems agrupados en la categoría de "contenido didáctico" cabe destacar que han sido valorados por los participantes de la investigación con valores altos, que oscilan entre 4,6 y 4,8 (Figura 4 ).

El ítem "el usuario recomendaría este programa a otros profesionales" recibe una puntuación alta de 4,8. A continuación le sigue en términos de mayor puntuación el ítem "el contenido ofrecido por el programa cumple las expectativas del consumidor", con un valor medio de 4,6. Por último, se sitúa el ítem "el programa ayuda a la orientación académica y profesional del alumnado", con una puntuación media de 4,3.

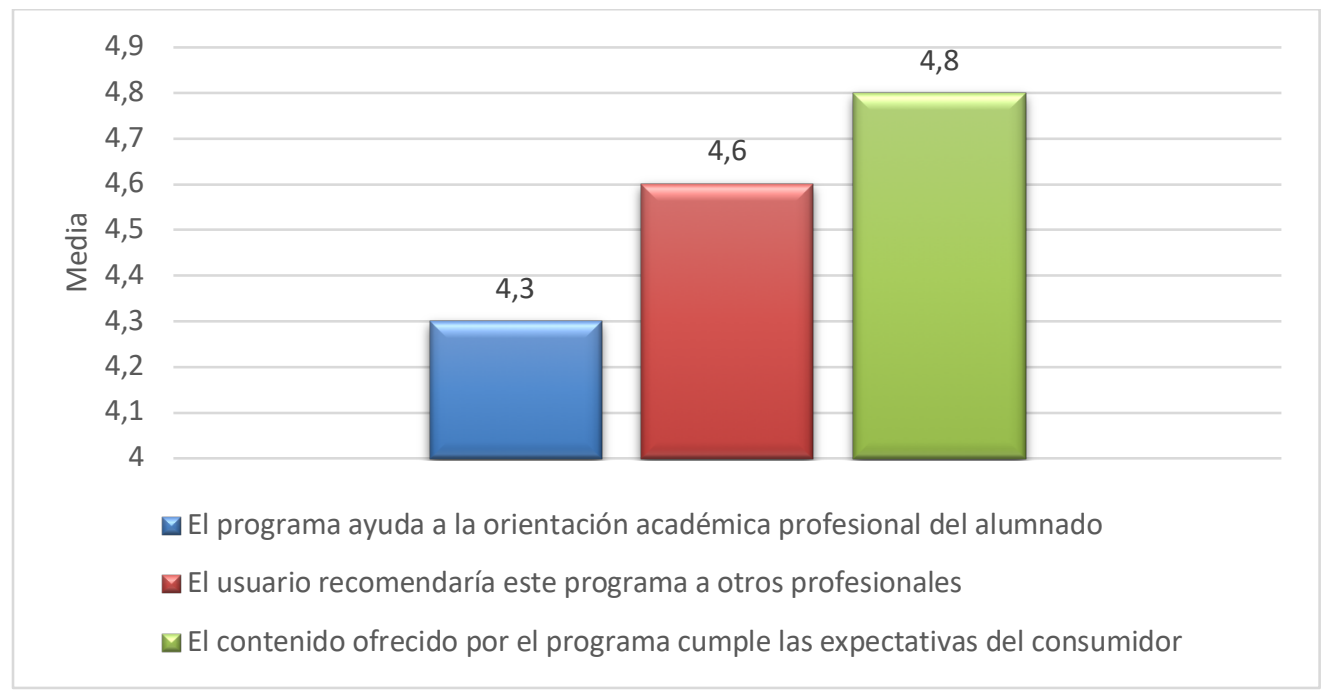

Figura 4. Gráfico: Apartado "Contenido didáctico" del cuestionario.

E) Gestión y producción de contenidos (material didáctico)

En cuanto a la gestión y producción de contenidos del programa, los orientadores participantes de la investigación han valorado tres ítems en esta categoría (Figura 5).

Ordenados de mayor a menor puntuación, se encuentra en primer lugar el ítem "compatibilidad del programa con todos los sistemas informáticos" con un valor máximo de 4,8 .

En segundo lugar en términos de mayor puntuación se encuentra el ítem "gestión de los ficheros y/o almacenamiento de material didáctico", con una puntuación media de 4,7.

En último lugar se encuentra el ítem "utilidad del diseño y producción de los contenidos", con un valor medio de 4,5 . 


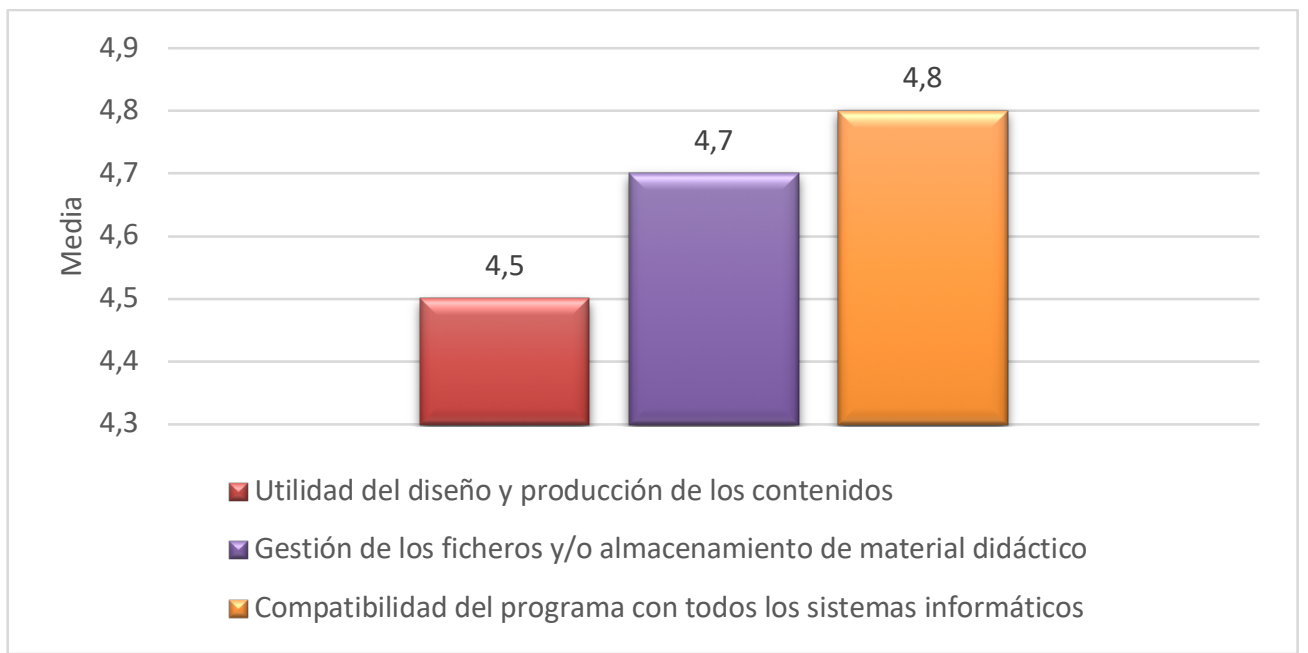

Figura 5. Gráfico: Apartado "Gestión y producción de contenidos" del cuestionario.

F) Evaluación y autoevaluación

En la última categoría del cuestionario, "evaluación y autoevaluación del programa", se han valorado dos ítems: "estadísticas/seguimiento de progresos" y evaluación del propio programa" (Figura 6). El primer ítem se refiere a la capacidad del programa para que el alumno pueda guardar los resultados de los cuestionarios que realiza y controlar su progreso y, por su parte, la capacidad del programa para realizar estadísticas de los perfiles profesionales que más se ajustan al alumnado a partir de dichos resultados obtenidos en los cuestionarios. El segundo ítem se refiere a la evaluación general que tienen los orientadores sobre el programa Orienta, es decir, qué opinión global les merece este programa.

En cuanto a las puntuaciones, el primer ítem, "estadísticas/seguimiento de progresos", recibe una valoración de 3,2 y el segundo ítem, "evaluación del propio programa", obtiene una puntuación de 4,7, próxima al valor máximo.

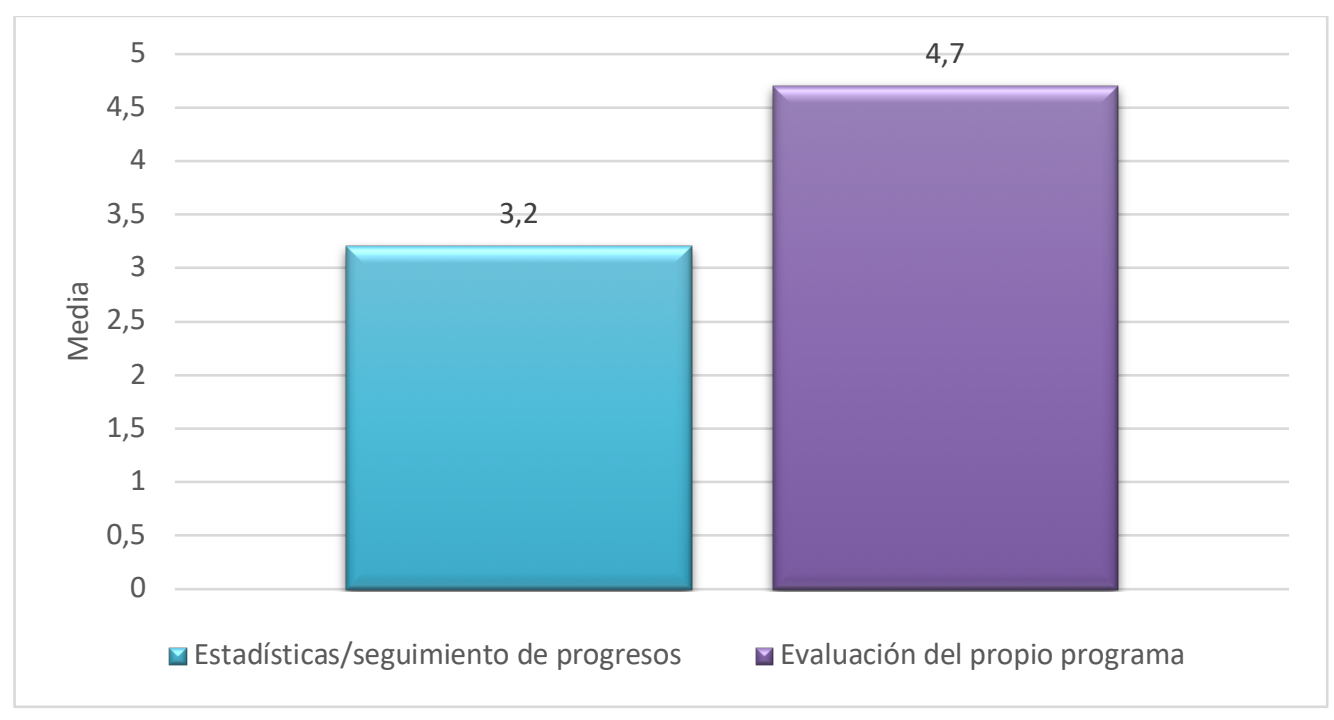

Figura 6. Gráfico: Apartado "Evaluación y autoevaluación" del cuestionario. 


\section{Discusión}

En este último apartado de esta investigación se tratará de exponer las conclusiones pertinentes de esta, y de presentar diferentes propuestas que puedan servir para mejorar posteriormente el programa Orienta. Este apartado se organiza en tres partes. Primero, se incluye un resumen de datos global, en el que se extraerán conclusiones a partir de los datos obtenidos a través de los cuestionarios. Segundo, tras observar los datos se examinarán diferentes propuestas de mejora del programa.

En primer lugar, la experiencia con la que contaban los orientadores educativos participantes ha resultado ser muy favorable de cara a los propósitos de la investigación, ya que la mayoría de los profesionales que participaron tenían entre un 1 y 5 años de experiencia (el $54 \%$ ). Como el programa Orienta se suele aplicar a lo largo del tercer trimestre del curso académico, se necesita que el profesional haya trabajado por lo menos durante un curso para poder estar en el centro educativo en el tercer trimestre y llevar a cabo el programa.

Otro aspecto que también ha resultado ser positivo es que la mayoría de los orientadores educativos que participaron en la investigación están trabajando en la actualidad en institutos de educación secundaria (76\%), frente a los que han desarrollado su labor profesional en equipos de orientación educativa (24 \%).

En cuanto a los propios resultados de los datos recogidos de los orientadores educativos sobre las valoraciones de las distintas categorías del programa Orienta han resultado ser, en general, bastante favorables.

Así, los datos recogidos del primer bloque de ítems del cuestionario, agrupados en la categoría de "aspectos generales" del programa Orienta, arrojan resultados muy positivos en su mayoría. Los aspectos del programa mejor valorados en esta categoría son:

- Facilidad de acceso a cualquier elemento de la plataforma.

- Orientación del usuario dentro del programa.

- Facilidad del uso del programa.

Por su parte, los ítems de esta categoría que han recibido puntuaciones menores y que, según la opinión de los orientadores participantes, habría que mejorar son los siguientes:

- Diseño gráfico.

- Organización de la pantalla.

- La estética del programa.

Por tanto, los aspectos que se podrían mejorar del programa son los relacionados con el diseño gráfico del mismo ya que, de acuerdo con los datos recogidos, la mayoría de los orientadores creen que los gráficos del programa Orienta podrían mejorarse para que este fuese más atractivo visualmente para los usuarios del programa.

En cuanto a las valoraciones del conjunto de ítems agrupados en la categoría de "comunicación" del cuestionario, son de media más bajas que las de la categoría de "aspectos generales". Los ítems que han recibido mejores puntuaciones en esta categoría son: 
- Guía del usuario/sistema de ayuda

- Blog del programa

Según las respuestas de los orientadores educativos, los aspectos que habría que mejorar del programa son:

- Calendario/agenda

- Tablón de anuncios

- Correo de contacto con el administrador/a

- Foro del programa

- Redes sociales

En conclusión, de acuerdo con las respuestas de los orientadores educativos, se puede deducir que la mayoría de los aspectos relacionados con la categoría de "comunicación" del programa Orienta estarían sujetas a mejoras.

En cuanto a las categorías de "contenido didáctico" y de "gestión y producción de contenidos", los resultados son muy favorables, ya que la media de las respuestas se sitúa cerca de la puntuación máxima. Los ítems agrupados dentro de estas categorías son los siguientes:

- El programa ayuda a la orientación académica profesional del alumnado.

- El usuario recomendaría este programa a otros profesionales.

- El contenido ofrecido por el programa cumple con las expectativas del consumidor.

- El diseño y producción de los contenidos son de mucha utilidad.

- Gestión de los ficheros y/o almacenamiento de material didáctico.

- Compatibilidad del programa con todos los sistemas informáticos.

En la última categoría del cuestionario, "evaluación y autoevaluación del programa, el segundo ítem, "Evaluación del propio programa" ha recibido una puntuación significativamente alta (4,7), mientras que el primer ítem "Estadísticas/seguimiento de progresos" ha obtenido una puntuación relativamente baja $(3,2)$, lo que significa que admitiría margen de mejora.

Tras haber extraído las conclusiones a partir de los datos globales, se procederá a continuación a reflexionar sobre los datos en relación con los objetivos generales y específicos planteados al inicio de esta investigación. Para ello, se hará a partir de los resultados y la discusión e interpretación de los datos obtenidos. Al final de este apartado, también se incluyen propuestas de mejora a partir de los ítems que han recibido puntuaciones bajas.

Los resultados encontrados demuestran que el programa Orienta es una herramienta TIC que tiene, en general, una valoración favorable en la mayoría de sus aspectos por los orientadores educativos.

Respecto a las propuestas de mejora del programa a partir de las conclusiones extraídas mediante los datos obtenidos a través de los cuestionarios se van a organizar entorno a propuestas de mejora de programa en relación con los aspectos: mejoras de tipo técnico, mejoras de tipo comunicativo, mejoras de los contenidos y mejoras de evaluación. 


\section{A) Mejoras de tipo técnico}

Este apartado está relacionado con la categoría de "aspectos generales" del formulario. En concreto, los aspectos mejorables técnicos se centran en cuestiones estéticas del programa, donde los datos señalaban que tanto el diseño gráfico, como la organización de la pantalla y la estética del programa podrían mejorarse para ser más atractivos visualmente y contribuir así a despertar la motivación e interés del alumnado.

En general, desde el punto de vista gráfico la interfaz es básica y el diseño del programa resulta simple y anticuado. Estos aspectos pueden hacer que la primera impresión que el alumnado se hace del programa sea desmotivante, unido al hecho de la escasa capacidad interactiva del programa y de la falta de recursos multimedia y audiovisuales. Por otro lado, el fondo utilizado en la pantalla principal resulta demasiado oscuro e impide ver claramente los elementos. Las categorías de dicha pantalla principal o menú parecen no seguir un orden lógico, y el usuario puede llevarse una primera impresión de que se encuentran desorganizadas. Se debe ir haciendo clic en cada elemento para "descubrirlo", en vez de poder verlo directamente sin necesidad de hacer clic o entrar en elemento. Carece, por tanto, de una visión global clara y adecuadamente organizada de los elementos que indique desde el primer momento qué se va a encontrar el usuario para poder acceder a la información o a los contenidos que busca más rápidamente.

En primer lugar, es llamativo el hecho de que todas las salidas y etapas educativas estén incluidas en la misma pantalla principal, independientemente de la etapa educativa del alumno (Bachillerato o Educación Secundaria Obligatoria), por lo que no resulta intuitivo y podría llevar a confusión. Por tanto, como primera mejora de propuesta en este sentido, la pantalla principal podría dividirse en dos subpantallas dependiendo de la etapa educativa en la que se encuentre el alumno. Cada subpantalla incluirá no solo los itinerarios disponibles, sino también todos los contenidos e información relevante de cada etapa (cuestionarios, becas, calendario con fechas clave, etc.).

En otro orden de ideas, los elementos del programa se podrían agrupar de otra forma. Por ejemplo, se entiende que las puertas de la portada principal representan simbólicamente las salidas o las vías profesionales. Así, "Universidad", "Otros estudios", "FP Superior", etc., se entienden como salidas. Sin embargo, los elementos "Información", "Títulos - Itinerarios" y "Cuestionarios" no son estrictamente salidas, sino información global que estaría en un plano diferente. Estas dos categorías, podrían aparecer en un aparte, en la esquina superior izquierda, por ejemplo, para captar la atención del usuario rápidamente. Además, como ya se mencionó anteriormente, cuando se accede a la pantalla principal (o a cada subpantalla, de llevarse a cabo la propuesta de mejora mencionada en el párrafo anterior), los ítems de cada categoría no se visualizan directamente, sino que se debe ir haciendo clic en cada uno. Esto, en última instancia, no resulta práctico y puede conllevar una pérdida de tiempo. Algunos elementos "no caben" en la pantalla y aparecen abreviados ("Si estás en Bachi.", "Preincripción Uni.", etc.). Esto, si bien no tiene por qué necesariamente dificultar la comprensión del usuario, al que se presupone conocedor del contexto, sí puede comprometer su comprensión de los ítems a primera vista cuando accede al programa.

En lo relativo a los recursos gráficos, se podrían reemplazar las imágenes o iconos existentes, poco atractivas, con otras más modernas. Además, la resolución de otras imágenes que pretenden ser informativas es de una calidad mejorable.

Por último, puesto que el programa contiene una cantidad enorme de información, puede resultar útil incorporar en el programa una barra de búsqueda para que tanto 
alumnos como orientadores y profesores localicen la información que les interesa fácilmente.

\section{B) Mejoras de tipo comunicativo}

Este apartado está relacionado con la categoría de "comunicación" del formulario. Específicamente, los aspectos que habría que mejorar en este apartado se centran en mejorar o incorporar herramientas para una comunicación efectiva de plazos, notas informativas y cualquier otra información de interés para el alumnado.

Como propuesta de mejora que se considera esencial por la utilidad que puede suponer para los alumnos, se propone añadir un apartado donde se incluya un calendario o una agenda con los diferentes plazos administrativos y otras fechas importantes. De esta forma, el alumnado tendrá a su disposición en un único apartado centralizado toda la información con respecto a los plazos importantes de inscripción, listados provisionales y definitivos, matriculación en los diversos estudios que quieran realizar e, incluso, fechas relacionadas con solicitudes de becas.

Otro aspecto que se sugiere que se incluya en el programa es que ya que la mayoría de los blogs de cada centro educativo no están actualizados o no tienen contenido relevante, se propone que en vez de utilizar esta herramienta, se cree un foro unificado para todos los orientadores que participan en el programa, donde se puedan intercambiar conocimientos, opiniones o experiencias de éxito, y de esta forma también simplificar los esfuerzos individuales en pro del beneficio de todos, dada la cantidad de información que es común a todos. Además, debido a la importancia que tienen las redes sociales para compartir información, se propone que se utilicen como recurso de ayuda. Por ejemplo, en la red social Twitter se puede acordar la utilización de un hashtag para compartir experiencias a través de este.

\section{C) Mejoras de los contenidos}

Este apartado se relaciona con la categoría "contenido didáctico" del cuestionario. Tal y como se observó a partir de los datos, por lo general, los orientadores participantes opinan que el contenido ofrecido por el programa cumple sus expectativas.

No obstante, dada la importancia del emprendimiento como salida, se considera que podría haber más información relativa a este ámbito, al menos incluso para que el alumnado se familiarice de una forma más directa con esta posibilidad. Por tanto, podría incluirse un apartado localizado dentro de la sección "Información", por ejemplo, titulado "Autónomos". Aquí podría incluirse información general sobre esta salida como trabajador por cuenta propia, como en qué consiste exactamente, cuáles son las condiciones, las ayudas comunitarias y estatales que se ofrecen, etc.

Por otro lado, también se echa en falta un apartado más general sobre las herramientas y recursos web para la búsqueda de empleo. Así, por ejemplo, se podría incluir un apartado con información sobre cómo elaborar currículums vítae, cartas de presentación, promoción de la marca personal en redes (en el caso de autónomos), enlaces clave a los sitios web de empleo, tanto de la comunidad autónoma (SAE) como del estado (SEPE), información sobre cursos para encontrar empleo o sobre los diferentes planes y proyectos de promoción de empleo entre los jóvenes como, por ejemplo, el programa de Garantía Juvenil. 


\section{D) Mejoras de evaluación}

Este apartado se corresponde con la categoría de "evaluación y autoevaluación del programa" del cuestionario. Según los orientadores educativos, el aspecto mejorable del programa Orienta se relaciona con las "Estadísticas/seguimiento de progresos".

Cabe destacar que sería muy útil para el alumnado poder crear una cuenta o perfil para que se pudiese guardar los resultados obtenidos en los diferentes cuestionarios de orientación a los que tienen acceso. De manera que una vez que hayan contestado los diferentes cuestionarios disponibles se puedan guardar estos datos en su perfil, y poder recuperarlos siempre que quieran. De esta manera el programa podría ofrecer poder crear un perfil para cada alumno donde se almacenen sus datos, y así poder acceder a ellos y recuperarlos siempre que los necesiten. De esta manera se puede hacer un seguimiento del progreso de cada alumno.

\section{Referencias}

Abad, D., Gago, S. y Merchante, C. (2019). Orientación Académica y Profesional: percepción del mercado de trabajo desde las Consejerías de Educación españolas. Educação e Pesquisa, 45

Aguaded, I., y Cabero, J. (2014). Avances y retos en la promoción de la innovación didáctica con las tecnologías emergentes e interactivas, Revista Educar, 67-83.

Fernández, E., Nogueira, M., y Couce, A. (2013). El uso de las Tecnologías de la Información y la Comunicación en la Orientación educativa: Explorando la familiaridad y la preparación de los profesionales del ámbito español, Revista Mexicana de Orientación educativa, 10(24), 45-57.

Hernández-Sampieri, R. y Mendoza, C. (2018). Metodología de la investigación: las rutas cuantitativa, cualitativa y mixta. México: Mc Graw-Hill.

Lozano, A. y Repetto, E. (2017). Las dificultades en el proceso de decisión vocacional en relación con: el género, el curso académico y los intereses profesionales, Revista Española de Orientación y Psicopedagogía, 18(1), 5-6.

Martínez, P., Pérez, F.J. y Martínez, M. (2014). Orientación Profesional en Educación Secundaria. Revista Electrónica Interuniversitaria de Formación del Profesorado, 17(1), 57-71.

McMillan, J.H. y Schumacher, S. (2005). Investigación educativa una introducción conceptual. Madrid: Pearson educación.
Muñoz, P. y González-Sanmamed, M. (2014). Posibilidades de la web 2.0 en orientación educativa: un estudio exploratorio sobre su presencia en las web de los departamentos de orientación de secundaria. Revista española de Orientación y psicopedagogía, 25(3), 36-55.

Prendes, M. (Dir.) (2009). Plataformas de campus virtuales de Software Libre: Análisis comparativo de la situación actual de las Universidades Españoles. Informe del proyecto EA-20080257 de la Secretaría de Estado de Universidades e Investigación.

Rodríguez, P., Ocampo, C. y Sarmiento, J. (2018). Valoración de la orientación profesional en la enseñanza secundaria postobligatoria, Revista de Investigación Educativa, 36(1), 7591.

Romero, C. y Montilla, M. (2015). La utilización de las TIC en la orientación educativa: un estudio exploratorio sobre la situación actual de uso y formación entre los profesionales de la orientación, Revista Española de Orientación y Psicopedagogía, 26(3), 78 - 95.

Sánchez, M. (Coord.). (2017). Orientación profesional y personal. Madrid: Universidad nacional de Educación a Distancia.

Tuero, E., Fernández-Castañón, A., García, M., García, J., y Bernardo, A. (2017). La transición a la universidad: eficacia de un programa de orientación en bachillerato apoyado en las TIC. Congresos CLABES. 


\section{Anexos}

\begin{tabular}{|c|c|c|c|c|c|c|c|}
\hline \multicolumn{8}{|c|}{$\begin{array}{l}\text { ANEXO I: Cuestionario de evaluación del programa Orienta para orientadores educativos } \\
\text { CUESTIONARIO DE EVALUACIÓN DEL PROGRAMA ORIENTA PARA ORIENTADORES EDUCATIVOS }\end{array}$} \\
\hline \multicolumn{8}{|l|}{ Datos del encuestado } \\
\hline \multicolumn{8}{|l|}{$\begin{array}{l}\text { Programa: Orienta } \\
\text { Trabaja en (marque con una X): } \\
\text { Instituto: } \quad \text { Equipo orientación educativa: } \\
\text { Años de experiencia como orientador: } \\
\end{array}$} \\
\hline \multicolumn{8}{|c|}{ Valore de 0 a 5 (siendo 0 muy malo y 5 excelente), cada uno de los siguientes aspectos generales de la plataforma. } \\
\hline Aspectos generales & 0 & 1 & 2 & 3 & 4 & 5 & $\begin{array}{l}\text { NS } \\
\text { /NC }\end{array}$ \\
\hline \multicolumn{8}{|l|}{ Facilidad de acceso a cualquier elemento del programa } \\
\hline \multicolumn{8}{|l|}{ Orientación del usuario dentro del programa } \\
\hline \multicolumn{8}{|l|}{ Diseño gráfico (colores, letra, iconos, etc.) } \\
\hline \multicolumn{8}{|l|}{ Organización de la pantalla } \\
\hline \multicolumn{8}{|l|}{ Estética del programa } \\
\hline \multicolumn{8}{|c|}{\begin{tabular}{|l|l|l|l|l|l|} 
Facilidad de uso del programa & & & & & \\
\end{tabular}} \\
\hline \multicolumn{8}{|c|}{ Valore de 0 a 5 (siendo 0 muy malo y 5 excelente), la funcionalidad de las siguientes utilidades del programa. } \\
\hline Comunicación & 0 & 1 & 2 & 3 & 4 & 5 & $\begin{array}{l}\text { NS } \\
\text { /NC }\end{array}$ \\
\hline \multicolumn{8}{|l|}{ Calendario/agenda } \\
\hline \multicolumn{8}{|l|}{ Tablón de anuncios } \\
\hline \multicolumn{8}{|l|}{ Guía de usuario/sistema de ayuda } \\
\hline \multicolumn{8}{|l|}{ Correo de contacto con el administrador } \\
\hline \multicolumn{8}{|l|}{ Foro del programa } \\
\hline \multicolumn{8}{|l|}{ Blog del programa } \\
\hline \multicolumn{8}{|l|}{ Uso de las redes sociales } \\
\hline Contenido didáctico & 0 & 1 & 2 & 3 & 4 & 5 & $\begin{array}{l}\mathrm{NS} \\
\text { /NC }\end{array}$ \\
\hline \multicolumn{8}{|l|}{ El programa ayuda a la orientación académica y profesional del alumnado. } \\
\hline \multicolumn{8}{|l|}{ El contenido ofrecido por el programa cumple las expectativas del consumidor. } \\
\hline \multicolumn{8}{|l|}{ El usuario recomendaría este programa a otros profesionales. } \\
\hline Gestión y producción de contenidos (del material didáctico) & 0 & 1 & 2 & 3 & 4 & 5 & $\begin{array}{l}\mathrm{NS} \\
/ \mathrm{NC}\end{array}$ \\
\hline \multicolumn{8}{|l|}{ Utilidad del diseño y producción de los contenidos. } \\
\hline \multicolumn{8}{|l|}{ Gestión de los ficheros y/o almacenamiento de material didáctico. } \\
\hline \multicolumn{8}{|l|}{ Compatibilidad del programa con todos los sistemas informáticos. } \\
\hline Evaluación y autoevaluación & 0 & 1 & 2 & 3 & 4 & 5 & $\begin{array}{l}\text { NS } \\
\text { /NC }\end{array}$ \\
\hline \multicolumn{8}{|l|}{ Estadísticas/seguimiento de progresos. } \\
\hline Evaluación del propio programa. & & & & & & & \\
\hline
\end{tabular}

